\title{
Regionalisierung und Dezentralisierung in Mittel- und Südosteuropa 1997-2007*
}

\author{
Michael W. Bauer • Diana Pitschel
}

Zusammenfassung: Der Beitrag zieht eine Bilanz der Ergebnisse der mittel- und südosteuropäischen Regionalisierungsforschung der vergangenen zehn Jahre. Das zentrale Ergebnis der Bestandsaufnahme ist, dass das wissenschaftliche Interesse an Erklärungsversuchen aus der Perspektive der Staatstransformation und der europäischen Konditionalitätspolitik, welche die Forschung bislang geprägt haben, nachlässt. Nachdem die Mehrzahl mittel- und südosteuropäischer Staaten der Europäischen Union beigetreten ist, gewinnt nun der Governance-Ansatz immer stärker an Bedeutung. Damit „normalisiert“ sich die Debatte über politische Dezentralisierung und Regionalisierung in Mittel- und Südosteuropa und konvergiert mit den einschlägigen Regionalisierungsdiskursen westeuropäischer Provenienz.

Schlagwörter: Regionalisierung $\cdot$ Mittel- und Südosteuropa $\cdot$ Systemtransformation EU-Konditionalität $\cdot$ Governance

\begin{abstract}
The review essay takes stock of the last decade of decentralisation and regionalisation research in Central and South Eastern Europe. Classifying the existing scholarship with regard to its focus of analysis, its explanatory programme, and methodological predilections, we suggest to distinguish three different agendas: system transformation, EU conditionality and subnational governance. We argue that scholarly interest in regionalisation and decentralisation issues from the perspective of state transformation or Europeanisation is vanishing. Instead, we witness the

* Der Aufsatz ist im Rahmen des DFG-Forschungsprojekts „Subnationale Governance-Präferenzen im Europäischen Mehrebenensystem“ entstanden. Wir danken dem Zukunftskolleg der Universität Konstanz für die finanzielle Unterstützung bei der Datenerhebung.
\end{abstract}

PD Dr. Michael W. Bauer $(\bowtie)$

Wissenschaftlicher Assistent

Lehrstuhl für Vergleichende Policy-Forschung und Verwaltungswissenschaft

Universität Konstanz, 78457 Konstanz

E-Mail: michael.w.bauer@uni-konstanz.de

Diana Pitschel

Wissenschaftliche Mitarbeiterin

Lehrstuhl für Vergleichende Policy-Forschung und Verwaltungswissenschaft

Universität Konstanz, 78457 Konstanz

E-Mail: diana.pitschel@uni-konstanz.de 
emergence of a subnational governance approach which is rooted in comparative politics and policy analysis. The debate about decentralisation and regionalisation in CEEC is thus in a process of "normalising" and converging with the Western European subnational political discourse.

Keywords: Regionalisation $\cdot$ Central and South Eastern Europe $\cdot$ Transformation • EU Conditionality $\cdot$ Governance

\section{Einführung}

Vor 1990 waren die kommunistischen Staaten im östlichen Europa rigoros zentralistisch organisiert, und ihre politischen Führungen konnten Forderungen nach regionaler und lokaler Emanzipation erfolgreich unterdrücken. Nach dem Niedergang des Kommunismus erwartete man, dass sich die bisher niedergehaltenen Regionalisierungs- und Dezentralisierungswünsche umgehend Bahn brechen würden. Jedenfalls nährten politische Absichtserklärungen zu Beginn der 1990er Jahre die Hoffnung auf die Einrichtung oder Wiederbelebung regionaler politischer Strukturen im gesamten östlichen Europa. In den folgenden Jahren konnte man denn auch die Schaffung regionaler oder den Ausbau lokaler politischer Strukturen in den mittel- und südosteuropäischen Ländern beobachten - wenn auch von durchaus unterschiedlicher Qualität. Angesichts dieser Dynamik auf der subnationalen Ebene überrascht es nicht, dass das Thema Dezentralisierung und Regionalisierung verstärkt in den Fokus der Osteuropaforschung rückte. Mittlerweile ist eine Vielzahl von Studien entstanden, die diese subnationalen Transformationsprozesse analysieren, ohne dass jedoch dieses Forschungsfeld als konsolidiert gelten könnte. Die Zeit scheint daher reif dafür, den sozialwissenschaftlichen Ertrag der jüngsten ,mittelund südosteuropäischen“ Dezentralisierungs- und Regionalisierungsforschung zu bilanzieren.

Konkretes Ziel des vorliegenden Beitrags ist es daher, die über das letzte Jahrzehnt entstandene sozialwissenschaftliche Forschungsliteratur zum Thema Dezentralisierung und Regionalisierung in Mittel- und Südosteuropa aufzuarbeiten und zu ordnen. Ein solcher Einordnungsversuch ist notwendig, weil die osteuropäische Regionalisierungsforschung sich zwar durch eine hohe Produktivität auszeichnet, aber der bislang erarbeitete theoretische Erkenntnisgewinn unklar bleibt. $\mathrm{Zu}$ viele Studien legen zu wenig Wert darauf, ihre Ergebnisse zu kontextualisieren und systematisch in Bezug zu anderen Forschungsresultaten zu bringen. Die Folge ist, dass unser deskriptives und theoretisches Verständnis von Regionalisierung und Dezentralisierung in den mittel- und südosteuropäischen Ländern weit hinter dem zurückbleibt, was aufgrund der regen Forschungstätigkeit in diesem Bereich zu erwarten wäre. Um keine Missverständnisse aufkommen zu lassen: Auch wir sind im Rahmen dieses Literaturberichts nicht in der Lage, die vorhandenen Defizite zu beheben. Allerdings wollen wir dazu beitragen, die Grundlage für eine - gerade auch konzeptionell - angezeigte Konsolidierung des Forschungsstandes zu schaffen. Dabei verfahren wir wie folgt: Zunächst sichten wir die einschlägigen wissenschaftlichen Arbeiten. Zum anderen klassifizieren wir die Studien nach ihrer Forschungsfrage, ihrem Erklärungsprogramm und ihrem methodischen Vorgehen. Vor diesem Hintergrund können wir Inhalte systematisieren und ein Ordnungsschema ent- 
wickeln, das für empirisch wie theoretisch interessierte Forscher hilfreich zu sein verspricht.

Der Aufsatz ist folgendermaßen gegliedert: Im nächsten Abschnitt werden die Kriterien vorgestellt, auf deren Grundlage die Auswahl der einschlägigen Regionalisierungsund Dezentralisierungsliteratur erfolgte. Danach wird ein kurzer Überblick über die Strukturen auf der subnationalen Ebene und die Hauptergebnisse subnationaler Reformen in den mittel- und südosteuropäischen Ländern gegeben. Im vierten und zentralen Teil entwickeln wir schließlich ein Ordnungsschema zur Klassifikation der existierenden Literatur. Unseres Erachtens können die verschiedenen Regionalisierungs- und Dezentralisierungsstudien drei unterschiedlichen Forschungsgebieten zugeordnet werden: der Systemtransformation, der Konditionalität und einer Governance-Perspektive. Wir kommen zu dem Ergebnis, dass das wissenschaftliche Interesse an Regionalisierung und Dezentralisierung im Kontext der Systemtransformation und der EU-Konditionalität schwindet. Stattdessen gewinnt der Governance-Ansatz, der eine dezidiert policy-analytische Perspektive einnimmt, immer stärker an Gewicht. Damit zeichnet sich eine „Normalisierung“ der Debatte um Dezentralisierung und Regionalisierung in den mittelund südosteuropäischen Ländern ab. Diese Normalisierung manifestiert sich darin, dass sich die Untersuchungsthemen der auf Mittel- und Südosteuropa fokussierten Forschung immer mehr den Diskursen und Fragestellungen der westeuropäischen Regionalismusdebatte annähern. Die Unterscheidung zwischen einer osteuropäischen und einer westeuropäischen Perspektive auf die Transformation subnationaler politischer Räume wird also künftig an Bedeutung verlieren. Insgesamt öffnen sich damit aus unserer Sicht neue und ertragreiche Möglichkeiten, die Prozessvielfalt und die Diversität der Regionalisierungsergebnisse sowie deren Auswirkungen auf die aktuelle Politikgestaltung in Mehrebenenkonstellationen vergleichend zu erforschen.

\section{Auswahl der einschlägigen Studien}

Vier Kriterien mussten erfüllt sein, damit eine Publikation in unsere Referenzliste aufgenommen werden konnte. Die ersten zwei sind offensichtlich. Es mussten Länderstudien sein, die einen oder mehrere empirische Fälle aus den unlängst der Europäischen Union beigetretenen Staaten Mittel- und Südosteuropas betrachten. Zweitens musste der empirische Fokus der Studien auf dem Wandel politischer Institutionen auf substaatlicher Ebene liegen. Ferner mussten die Studien in der letzten Dekade und auf Englisch erschienen sein. Diese Suchkriterien ergaben sich aus den folgenden Überlegungen.

Die Termini „Mittel- und Osteuropa“, „Südosteuropa“ oder „,mittel- und osteuropäische Länder“ etc. werden in der Literatur uneinheitlich verwendet. ${ }^{1}$ Um die zu erstel-

1 Die meisten Studien beziehen sich implizit oder explizit auf die mittel- und südosteuropäischen Länder, die mittlerweile der EU beigetreten sind, so z. B. Bachtler et al. (2000); Brusis (2002); Frenkel/Nickel (2005); Grabbe (2001); Hughes et al. (2004a); Mudde (2005); O'Dwyer (2006); Schimmelfennig/Sedelmeier (2004, 2005). Andernorts wird der Begriff Mittel- und Osteuropa häufig recht großzügig definiert (und schließt Staaten ein, die an anderer Stelle zu Südosteuropa gerechnet werden). Zum Beispiel betrachtet die OECD Albanien, BosnienHerzegowina, Bulgarien, Kroatien, die Tschechische Republik, Estland, Ungarn, Lettland, 
lende Liste von Referenztexten dennoch sinnvoll eingrenzen zu können, wurden die Arbeiten zur Regionalisierung und Dezentralisierung für jene Staaten Mittel- und Südosteuropas gesucht, die bis zum gegenwärtigen Zeitpunkt der Europäischen Union beigetreten sind. ${ }^{2}$ Damit nehmen wir in Kauf, dass die Auswahl der Studien von einem gewissen „EU-Bias“ geprägt ist. Ein solcher ist jedoch kaum vermeidbar, da es praktisch keine einschlägigen Studien zur Regionalisierung und Dezentralisierung in den mittel- und südosteuropäischen Ländern gibt, die nicht Bezug auf den europäischen Integrationsprozess nehmen.

Um als Referenztext in die Literaturanalyse aufgenommen zu werden, war eine weitere grundlegende Bedingung, dass sich die Arbeiten explizit auf die intermediäre Regierungsebene konzentrieren, die wir als direkt unterhalb der nationalen, aber über der lokalen Ebene gelegene „subnationale“ Ebene definieren. Diese Abgrenzung kann kontrovers diskutiert werden. ${ }^{3}$ Ihre relative Offenheit scheint aber gerade auch deshalb angebracht, da ja möglichst die gesamte Breite der bestehenden intermediären politischen Strukturen in Mittel- und Südosteuropa in den Blick genommen werden soll. ${ }^{4}$

Zudem soll die einschlägige Forschungsleistung innerhalb eines bestimmten Zeitraums analysiert werden. Um in unsere Liste aufgenommen $\mathrm{zu}$ werden, musste das Publikationsdatum einer Studie zwischen 1997 und 2007 liegen. Der Grund für diese Eingrenzung ist ein einfacher: In diesem Zeitraum wurden die wesentlichen Reformen der subnationalen politischen Strukturen in den mittel- und südosteuropäischen Ländern beschlossen und implementiert (siehe Tabelle 3, S. 136).

Schließlich müssen unsere Referenztexte auf Englisch publiziert worden sein. Der Grund hierfür ist das Anliegen, die internationale Debatte in den Blick zu nehmen. Diese wird mittlerweile fast ausschließlich in englischsprachigen Zeitschriften und Monografien geführt. Man könnte hier einwenden, dass der angestrebte Forschungsüber-

Litauen, Mazedonien, Polen, Rumänien, Slowakei, Slowenien und Jugoslawien (Serbien und Montenegro) als MOE-Länder. Der Begriff Südosteuropa wird zumeist topografisch definiert und bezieht sich auf die Staaten der Balkanhalbinsel, des Pannonischen Beckens und des transkarpathischen Raumes, d. h. Albanien, Bosnien und Herzegowina, Bulgarien, Griechenland, Kroatien, Mazedonien, Moldawien, Montenegro, Rumänien, Serbien, Slowenien, Ungarn und Türkei (siehe Ständiger Ausschuss für geographische Namen). Um Missverständnisse zu vermeiden, definieren wir die in unserem Aufsatz berücksichtigte Auswahl an Ländern als „,mittel- und südosteuropäische Länder“.

2 Das sind Bulgarien, Estland, Lettland, Litauen, Polen, Rumänien, Slowakei, Slowenien, die Tschechische Republik und Ungarn.

3 Der Begriff der Dezentralisierung wird gewöhnlich nicht nur für den Transfer politischer Autorität auf die regionale, sondern auch auf die lokale Regierungsebene verwendet. In diesem Beitrag muss die lokale Dimension der Dezentralisierung jedoch außen vor bleiben. In den Fällen, in denen wir in einem Land mehr als eine Ebene zwischen der nationalen und der lokalen Ebene vorfinden, fokussieren wir zudem auf jene Regionalebene, die eine Exekutive umfasst, deren verantwortliches Personal sich allgemeinen Wahlen stellen muss. In Ländern, in denen es keine solche gewählten Exekutiven oder Versammlungen auf der regionalen Ebene gibt, weichen wir auf die höchste subnationale Ebene der dezentralisierten Verwaltung als empirischen Untersuchungsgegenstand aus.

4 Hierfür wäre beispielsweise der gängigere, aber letztendlich politisch aufgeladene Begriff ,regional“" wesentlich weniger geeignet (Keating 1998: 9-10). 
blick auch die wichtigsten landessprachlichen Publikationen enthalten sollte, selbst wenn diese nicht auf Englisch erschienen sind. Das ist grundsätzlich sicher richtig, wenn auch angesichts der hierfür notwendigen Sprachkompetenz praktisch schwierig umzusetzen. Es gilt aber zu bedenken, dass bedeutsame nationale Forschungsergebnisse gewöhnlich, wenn auch etwas zeitversetzt, in internationalen Zeitschriften präsentiert werden. Wir haben diese Überlegung nachgeprüft und für ausgewählte Experten personenbezogen recherchiert. Obwohl, so unser Ergebnis, eine gewisse Verzerrung zugunsten englischsprachig publizierter Studien nicht ausgeschlossen werden kann, ist das Risiko der Vernachlässigung wichtiger Forschungsergebnisse bei dem gewählten Vorgehen minimal.

Bei der Auswahl der zu berücksichtigenden Publikationen haben wir im ersten Schritt die bekannten Datenbanken wie den Social Science Citation Index (SSCI) nach relevanten Studien durchsucht. Überraschenderweise hat diese Suche nur sehr wenige Ergebnisse geliefert: Viele aus der Sicht der Dezentralisierungs- und Regionalisierungsforschung einschlägige Publikationen sind nicht im SSCI gelistet. ${ }^{5}$ Da eine solche herkömmliche Literaturrecherche in unserem Sinne also wenig zielführend war, haben wir eine Liste politikwissenschaftlicher Zeitschriften zusammengestellt, die entweder ihren Fokus auf die mittel- und südosteuropäischen Länder oder auf subnationale Entitäten allgemein ausgerichtet haben, oder die - wie das Journal of European Public Policy oder das Journal of Common Market Studies - regelmäßig einschlägige Aufsätze zum Thema Dezentralisierung und Regionalisierung in den mittel- und südosteuropäischen Ländern publizieren (siehe Tabelle 1).

Tabelle 1: Liste der ausgewerteten Zeitschriften, 1997-2007

- Central European Political Science Review

- Communist and Postcommunist Studies

- Comparative European Politics

- Comparative Political Studies

- East European Politics and Society

- Europe-Asia Studies

- European Journal of Political Research

- European Urban and Regional Studies

- Governance: An International Journal of Policy, Administration and Institutions

- International Journal of Urban and Regional Research

- Journal of Common Market Studies

- Journal of European Integration

- Journal of European Public Policy

- Perspectives on European Politics \& Society

- Regional and Federal Studies

Diese Zeitschriften haben wir dann systematisch ausgewertet. Zur Konsolidierung unserer Suchergebnisse haben wir zusätzlich eine Stichwortsuche mit den Stichwortkombinationen „decentralisation“/,decentralization“ AND „Central and Eastern Europe“,

5 Der Hauptgrund dafür ist, dass die meisten politikwissenschaftlichen Zeitschriften, die zu Regionalisierung und Dezentralisierung in MOE publizieren, nicht im SSCI berücksichtigt sind. 
„regionalisation“/,regionalization“ AND „Central and Eastern Europe“ ebenso wie „regional policy“ AND „Central and Eastern Europe“ im WorldCat-Katalog durchgeführt. Abschließend haben wir unsere Suchergebnisse mithilfe des „Schneeballverfahrens“ ergänzt. Hierzu haben wir Bibliografien bereits identifizierter Aufsätze nach Hinweisen zu weiteren einschlägigen Arbeiten durchgesehen. Insgesamt haben wir in unserer Recherche auf diese Weise 65 einschlägige Monografien (einschließlich herausgegebener Bände) und Aufsätze identifizieren können. Diese Publikationen sind mit einem * gekennzeichnet und im Literaturverzeichnis am Ende dieses Artikels gelistet.

\section{Die Reform subnationaler politischer Strukturen in den mittel- und südosteuropäischen Ländern - Zeitrahmen und Ergebnisse}

Nach 1990 wurden in allen Staaten Mittel- und Südosteuropas subnationale politische Einheiten und lokale Selbstverwaltungen neu geschaffen, wiederbelebt oder reformiert. Zudem haben die Beitrittsstaaten ihr Territorium nach den statistischen Regionalisierungsstandards der EU $\left(\mathrm{NUTS}^{6}\right)$ gegliedert, um im Rahmen der europäischen Kohäsionspolitik gewährte Finanzhilfen und Strukturmittel optimal in Anspruch nehmen zu können. Auch wenn dieser Trend in allen mittel- und südosteuropäischen Ländern gleichermaßen zu beobachten war, variieren die Ergebnisse dieser Institutionenbildung von Land zu Land. Um die Komplexität unterschiedlicher subnationaler Strukturen besser erfassen zu können, bietet es sich an, zum einen zu unterscheiden, wie viele subnationale politische Ebenen in einem Land existieren, und zum anderen zu differenzieren, ob es sich dabei jeweils um eine dekonzentrierte Verwaltungsebene des Zentralstaates oder tatsächlich um eine (wenigstens in Teilen) eigenständige politische Ebene im subnationalen Raum handelt. Eine Übersicht über Zeitrahmen und Ergebnisse der Transformation subnationaler politischer Räume der mittel- und südosteuropäischen Länder findet sich in Tabelle 2. Demnach verfügen die meisten Länder über nur eine intermediäre, zwischen der lokalen und der nationalen angesiedelte Ebene. Einige andere verfügen zusätzlich über einen Zusammenschluss mehrerer lokaler Akteure, also eine Art Kreisebene, die aber eigentlich der lokalen Selbstverwaltung zuzuordnen ist. Eine weitere Gruppe besitzt sowohl Strukturen subnationaler Selbstverwaltung als auch dekonzentrierte nationale Regierungsorgane.

Tabelle 3 gibt einen Überblick über diejenigen subnationalen Einheiten, die die empirische Grundlage unserer Sekundärauswertung bilden. Die Tabelle zeigt, wann diese Entitäten geschaffen wurden und die Anzahl der Studien, die jeweils empirisch Bezug auf die betreffenden subnationalen Einheiten nehmen. Die Tabelle bildet einen Trend in der mittel- und osteuropäischen Regionalisierungsforschung ab. Während sich die große Mehrheit der Studien auf die ,großen“ Staaten Polen, Tschechische Republik, Ungarn und Slowakei konzentriert, gibt es nur wenige Arbeiten, die die drei baltischen Staaten sowie Rumänien, Bulgarien und Slowenien in Bezug auf Fragen der Dezentralisierung und Regionalisierung in den Blick nehmen.

6 Das Akronym NUTS steht für „Nomenclature des unités territoriales statistiques“. 
Tabelle 2: Ergebnisse der Reform subnationaler politischer Räume in den mittel- und südosteuropäischen Ländern 1997-2007

\begin{tabular}{|c|c|c|c|}
\hline Ebene & Statistische & \multicolumn{2}{|c|}{ Struktur der subnationalen Exekutive $^{8}$} \\
\hline National & \multirow{3}{*}{$\begin{array}{l}\text { NUTS } 0 \\
\text { NUTS } 1-3\end{array}$} & \multicolumn{2}{|l|}{ Nationale Regierung $^{9}$} \\
\hline Subnational & & \multirow{2}{*}{$\begin{array}{l}\text { Dezentralisierte } \\
\text { nationale Regierung } \\
\text { BG, EE, HU, LT, LV, } \\
\text { PL, RO, SK }\end{array}$} & $\begin{array}{l}\text { Subnationale Regierung } \\
\text { PL }\end{array}$ \\
\hline Subnational & & & $\begin{array}{l}\text { Subnationale Selbstverwaltung } \\
\text { (Bezirk/Kreis) } \\
\mathrm{CZ}, \mathrm{HU}, \mathrm{RO}, \mathrm{SK},[\mathrm{SI} / \mathrm{PL}]\end{array}$ \\
\hline Lokal & LAU $^{10} 1-2$ & Lokale Selbstverwaltung & (ähnlich in allen Ländern) \\
\hline
\end{tabular}

Tabelle 3: Subnationale politische Räume in den mittel- und südosteuropäischen Ländern, zentrale Reformen und wissenschaftliche Rezeption

\begin{tabular}{lllc}
\hline Land & $\begin{array}{l}\text { Subnationale Einheit } \\
\text { (Name, Anzahl) }\end{array}$ & $\begin{array}{l}\text { Wichtigste subnationale Reformen } \\
(\mathbf{J a h r})^{\mathbf{1 1}}\end{array}$ & $\begin{array}{c}\text { Zahl der Studien in } \\
\text { unserem Sample }\end{array}$ \\
\hline Bulgarien & oblast, 28 & 1999 & 7 \\
Tschechische & kraj, 14 & 2000 & 24 \\
Republik & & [einige, zuletzt] 2005 & 9 \\
Estland & maakonnad, 15 & 1999 & 24 \\
Ungarn & megye, 19 & 1998 [anhaltende Reformdiskussionen] & 5 \\
Lettland & rajon, 26 + 7 & 1998, 2000, 2003 & 3 \\
Litauen & apskritys, 10 & 1999 & 31 \\
Polen & województwo, 16 & 1998, 2004 & 8 \\
Rumänien & județ, 42 & 2001 & 19 \\
Slowakei & samoprávny kraj, 8 & 1998 [anhaltende Reformdiskussionen] & 7 \\
Slowenien & pokrajina, 14 [geplant] & 12 \\
Allgemein & & & 7 \\
\hline
\end{tabular}

Quelle: Bailey/De Propris (2002), Brusis (1999, 2002, 2003), CoR (2005), Keating/Hughes (2003), EZFF 2004-2007); eigene Zusammenstellung.

7 Wir danken Ansgar Schäfer für den Entwurf dieser Tabelle.

$8 \mathrm{BG}=$ Bulgarien, $\mathrm{CZ}=$ Tschechische Republik, $\mathrm{EE}=$ Estland, $\mathrm{HU}=$ Ungarn, $\mathrm{LT}=$ Litauen, $\mathrm{LV}=$ Lettland, $\mathrm{PL}=$ Polen, $\mathrm{RO}=$ Rumänien, $\mathrm{SI}=$ Slowenien, $\mathrm{SK}=$ Slowakei.

9 In Polen, der Tschechischen Republik und Rumänien verfügt die subnationale Ebene über die zweite Kammer im nationalen Parlament über zusätzliche Einflussnahmemöglichkeiten.

10 Die Abkürzung LAU steht für Lokale Verwaltungseinheit.

11 Die Literatur liefert diesbezüglich widersprüchliche oder unspezifische Daten. Folglich handelt es sich bei den hier angegebenen Jahreszahlen entweder um das Datum der Einführung der gegenwärtig bestehenden subnationalen Strukturen oder das Datum der Verabschiedung der jeweiligen gesetzlichen Grundlage.

12 Wenn eine Studie sich auf subnationale Einheiten verschiedener Länder bezieht, wird sie für jedes untersuchte Land gezählt. In diese Auswertung sind alle „Primärquellen“ eingeflossen, die mit einem * markiert sind. 


\section{Systemtransformation, Konditionalität und Governance: die drei Forschungsprogramme der Regionalisierungsforschung}

Im Rahmen der Analyse der Literatur über Regionalisierung und Dezentralisierung in den mittel- und südosteuropäischen Ländern haben wir drei unterschiedliche Forschungsprogramme bzw. Ansätze identifiziert. Obwohl es aufgrund des engen thematischen Fokus natürlich Überschneidungen gibt, können diese drei Programme doch relativ klar nach ihren jeweiligen zentralen Forschungsfragen, den entwickelten Erklärungsmechanismen und dem jeweiligen methodischen Vorgehen unterschieden werden. Der erste Ansatz betrachtet Regionalisierungs- und Dezentralisierungsprozesse in den mittel- und südosteuropäischen Ländern als eine Dimension der Systemtransformation nach dem Zusammenbruch des Kommunismus. Im Kern des zweiten Forschungsprogramms steht die Schaffung bzw. Wiederbelebung subnationaler institutioneller Strukturen unter dem Druck des „Konditionalitätsregimes“ der EU. Der dritte, gegenwärtig Kontur gewinnende Forschungsansatz betrachtet Dezentralisierung und Regionalisierung im Kontext des Wandels von Government zu Governance.

Abbildung 1: Die Forschungsschwerpunkte der mittel- und südosteuropäischen Dezentralisierungs- und Regionalisierungsforschung, 1997-2007

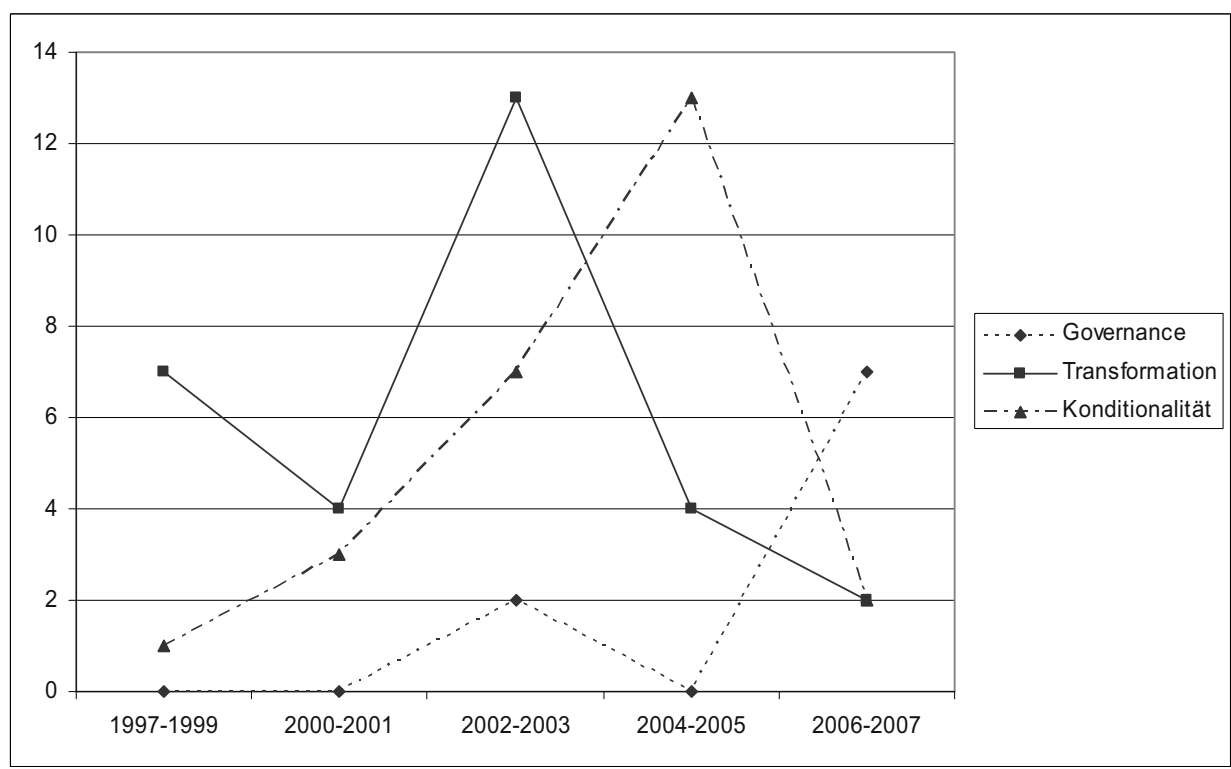

Quelle: eigene Zusammenstellung. Die Datenpunkte geben die Anzahl der veröffentlichten Studien, geordnet nach Forschungsschwerpunkt und Erscheinungszeitraum, wieder.

Das Schaubild liefert einen Eindruck von der Produktivität der einzelnen Forschungsprogramme im Zeitverlauf. Bis 2003 wurden hauptsächlich Studien publiziert, die die subnationale Dynamik in Mittel- und Südosteuropa im Zusammenhang mit der Transformation der ehemals kommunistischen Systeme betrachten. Mit der Konkretisierung 
der Beitrittsperspektive ab 2002 richtete sich der Fokus der Regionalisierungs- und Dezentralisierungsforschung verstärkt auf die Rolle der EU und ihrer Konditionalitätspolitik. Insbesondere zwischen 2004 und 2005 ist die Anzahl der Publikationen, die sich mit den Auswirkungen des EU-Konditionalitätsregimes auf die subnationalen Strukturen in Mittel- und Südosteuropa befassen, stark angestiegen. Seit dem Abschluss der Osterweiterung sind zunehmend Fragen subnationaler Governance, die vor 2004 praktisch keine Rolle gespielt haben, auf die Agenda der mittel- und südosteuropäischen Regionenforschung gerückt. Seit 2006 machen Governance-bezogene Studien den Großteil der einschlägigen Publikationen im Forschungsfeld aus; die Anzahl der Transformations- und Konditionalitätsstudien nimmt hingegen ab.

\subsection{Systemtransformation}

Studien, die dem Systemtransformations-Ansatz zugeordnet werden können, verorten die Dezentralisierung und Regionalisierung in den mittel- und südosteuropäischen Ländern im Kontext der Transformation dieser Staaten von kommunistischen Regimen hin zu demokratischen Staaten. Sie nehmen in der Regel eine retrospektive Sichtweise ein und untersuchen die spezifischen Bedingungen sowie die Resultate subnationaler Reformen (Bachtler et al. 2000; Batt 2002a, 2002c, 2003; Fowler 2002; Gorzelak 1998; Illner 2002; Kirchner 1999; Tatur 2004; Wolczuk 2002).

Diese Studien spiegeln die enormen Diskrepanzen zwischen den anfänglich sehr hohen Erwartungen einerseits und den tatsächlichen Resultaten der Reformen auf der subnationalen Ebene andererseits wider. Sowohl in der Wissenschaft als auch in der Praxis herrscht weitgehender Konsens dahingehend, dass Dezentralisierung und Regionalisierung wichtige Schritte dieser Staaten in Richtung Demokratisierung darstellen (Gerner 2005; Hughes et al. 2002; Wolczuk 2002). Insbesondere für die historischen Regionen schien sich mit dem Zusammenbruch der kommunistischen Regime ein Gelegenheitsfenster zu öffnen, mehr Autonomierechte zu erhalten. In Anbetracht dieser „Regionalisierungseuphorie" stellt sich jedoch die Frage, warum die Reformen in den meisten Fällen keine politisch stärkeren subnationalen Einheiten hervorgebracht haben. Die Systemtransformationsliteratur vertritt die These, dass im Wesentlichen zwei Faktoren für die politische Schwäche regionaler politischer Strukturen in Mittel- und Südosteuropa verantwortlich sind: zum einen die besondere Geschichte dieser Region und zum anderen die Angst der politischen Eliten in den neu entstehenden Demokratien vor einer Destabilisierung durch zentrifugale Kräfte. Analysen aus der Mitte dieses Forschungsprogramms konnten zeigen, dass föderale Ideen und die Verteilung politischer Macht auf der vertikalen Achse historisch nur eine sehr untergeordnete Rolle in den mittel- und südosteuropäischen Ländern gespielt haben. Schon allein aus diesem Grund sei eine Etablierung starker subnationaler Einheiten mit weitreichenden Kompetenzen kaum zu erwarten gewesen (Mastny 2000; Pieper 2006; Ortino et al. 2005). Alarmiert durch die schicksalhaften Entwicklungen in der Tschechoslowakei und in Jugoslawien nach 1990 begegneten viele politische Akteure zudem der Idee eines Transfers nationaler Gestaltungsmacht auf nachgeordnete Ebenen ohnehin mit zunehmender Skepsis. Darüber hinaus werden Animositäten über die - teilweise arbiträren - Grenzziehungen nach dem Ersten und Zweiten Weltkrieg zwischen diesen Staaten, die sich bis in die Gegenwart fortset- 
zen, angeführt, um zu erklären, warum sich Politiker weigern, „Regionen“ mit weitreichenden Autonomierechten auszustatten. Das ist insbesondere dort der Fall, wo die Befürchtung besteht, dass Minderheiten eines Staatsgebiets mit ihren ethnischen „Brüdern und Schwestern", die auf der anderen Seite der Staatsgrenze die Mehrheitsbevölkerung bilden, enger als politisch opportun zusammenarbeiten wollen. Dadurch wird deutlich, dass die politische Konsolidierung auf der zentralstaatlichen Ebene in den Zeiten der Systemtransformation in den mittel- und südosteuropäischen Ländern Priorität vor Dezentralisierungs- und Regionalisierungsbestrebungen hatte (Wolczuk 2002; Bessenyey Williams 2007; Tägil 1999).

Zur Erklärung der Regionalisierungsdynamik im Transformationskontext wird theoretisch zumeist auf Mechanismen Bezug genommen, die dem historischen Erbe geschuldet sind (legacies). Die einschlägigen Studien orientieren sich dabei am Programm des historischen Institutionalismus. Man nimmt an, dass sich historische Konstellationen auf das gegenwärtig bestehende institutionelle Gefüge auswirken, dass politische Strukturen also pfadabhängig sind und sich selbst reproduzieren (Hall/Taylor 1996; Immergut 1998; Thelen/Steinmo 1992). So demonstriert eine Reihe von Studien, dass gerade die Verwaltungsstrukturen aus der Zeit vor dem Ersten Weltkrieg eine wichtige Modellfunktion für die jüngsten Reformen der subnationalen Regierungsebenen in der Tschechischen Republik, in Ungarn und in Polen hatten. Alle drei Staaten haben beispielsweise das dualistische Verwaltungsmodell österreichisch-ungarischer Prägung, das dort bis Ende des Ersten Weltkriegs existierte, nach dem Fall des Kommunismus wieder eingeführt (Brusis 1999: 7; Pieper 2006).

So plausibel die zentralen Argumente auch sind, die im Kontext dieses Ansatzes entwickelt wurden, Zweifel und Unklarheiten bleiben. So ist es beispielsweise schwierig, die Ergebnisse einzelner Studien derart nutzbar zu machen, dass analytisch fundierte Aussagen darüber möglich werden, welche legacies - jene aus der Kaiserzeit, aus der Zwischenkriegszeit oder aus der kommunistischen Vergangenheit - sich in Bezug auf konkrete politische Entscheidungen in der Gegenwart durchsetzen.

Was die methodologischen Präferenzen der Transformationsstudien anbelangt, ist eine klare Vorherrschaft historischer und kulturell-anthropologisch geprägter, qualitativer Studien feststellbar. Dabei findet man hauptsächlich individuelle Länderstudien, während die Anzahl der Arbeiten mit einem vergleichenden Design gering ist. Darüber hinaus sind überdurchschnittlich häufig historische Regionen wie das Banat, Schlesien, Mähren oder Pommern Gegenstand empirischer Untersuchungen (Bafoil 1999; Batt 2002a, 2002b; Bialasewicz 2002; Wolczuk 2000; Yoder 2003). Während sich die Betrachtung dieser Regionen einerseits anbietet, um mit Referenz zum historischen Institutionalismus den Einfluss historischer legacies zu testen, setzt man sich andererseits mit dieser einseitigen Fokussierung und der Vernachlässigung von weniger historisch determinierten Vergleichsfällen der Gefahr des argumentativen Zirkelschlusses aus. Auch ist die bevorzugte Untersuchung der subnationalen Dynamik in den größeren mittel- und südosteuropäischen Ländern aus empirisch-pragmatischen Gründen verständlich. Dennoch bleibt so das Bild der subnationalen Reformprozesse in Mittel- und Südosteuropa im Transformationskontext durch den Mangel an Studien zu den kleineren Staaten, aber auch zu Bulgarien oder Rumänien unvollständig. Trotz dieser Kritik ist die Leistung des Transformationsansatzes offensichtlich. Denn es wurde ein Grund- 
stock an systematischem Wissen über die politischen Prozesse und Ergebnisse von Regionalisierung und Dezentralisierung in den mittel- und südosteuropäischen Ländern während der 1990er Jahre gelegt. Damit bilden diese Studien den Ausgangspunkt für alle weiteren Forschungsanstrengungen im Hinblick auf Regionalisierung und Dezentralisierung in Mittel- und Südosteuropa.

\subsection{EU-Konditionalität}

Nachdem die EU-Beitrittsverhandlungen mit den mittel- und südosteuropäischen Ländern aufgenommen hatte und die Europäische Kommission den Kandidaten finanzielle Mittel zur Unterstützung bei der notwendigen Umsetzung des acquis communautaire des Rechtsbestandes der EU - hatte anbieten können, rückte die Frage nach dem Einfluss der Europäischen Union auf die nationale Politik und den Aufbau institutioneller Strukturen in den mittel- und südosteuropäischen Ländern in den Mittelpunkt. Mit dieser Neuausrichtung des analytischen Fokus konsolidierte sich ein zweiter Forschungsansatz, der die einsetzende regionale Dynamik vornehmlich im Kontext der Vorbereitungsbeihilfen der EU und von deren Konditionalitätsregime analysierte.

Obwohl der durch den Transformations- und den Konditionalitätsansatz fokussierte Zeitrahmen nahezu identisch ist, ist offensichtlich, dass sich das Konditionalitätsprogramm weniger für die Voraussetzungen oder Kontextfaktoren der Regionalisierung in den mittel- und südosteuropäischen Ländern interessiert. Vielmehr geht es darum, Aufschluss darüber zu gewinnen, wann, wie und unter welchen Bedingungen die Europäische Union Einfluss auf die Schaffung subnationaler Institutionen ausgeübt hat. Im Rahmen des Konditionalitätsansatzes wird neben innerstaatlichen Faktoren und nationalen historischen legacies also die EU selbst als zentrale Erklärungsvariable eingeführt. Das wesentliche Ziel einschlägiger Studien liegt zum einen in der Identifizierung der Konstellationen, die die Interaktion zwischen EU und innerstaatlichen Faktoren determinieren; man will also unterschiedliche Intensitäten von EU-Einfluss im Sinne von Europäisierungsmustern herausarbeiten. Ferner soll geklärt werden, unter welchen Bedingungen der Einfluss nationaler Faktoren einerseits oder der EU andererseits stärker auf die Ausgestaltung subnationaler Strukturen wirkt (Batt 2003; Brusis 2002, 2003, 2005; Czernielewska/Paraskevopoulos/Szlachta 2004; Ferry/McMaster 2005; Hughes et al. 2004a, 2000b; Keating/Hughes 2003; Marcou 2002; O’Dwyer 2006; Paraskevopoulos/ Leonardi 2004; Sturm/Dieringer 2005; Szemlér 2000).

Die im Kontext des Konditionalitätsansatzes entwickelten Argumente sind im Wesentlichen aus den Europäisierungstheorien abgeleitet, die sich bis dato lediglich auf die zentrale Staatsebene der Mitgliedstaaten der Europäischen Union beschränkt hatten. Das primäre Interesse dieser Europäisierungstheorien liegt auch bei der Untersuchung subnationaler Prozesse auf den Auswirkungen des europäischen Integrationsprozesses auf die institutionellen Strukturen und Akteursstrategien innerhalb der Mitgliedstaaten. Die grundlegende Annahme in Bezug auf die Beitrittskandidaten ist dabei, dass der transformatorische Einfluss der EU auf die nationale Politik umso stärker wird, je näher der Beitrittstermin rückt (Hix/Goetz 2000; Schimmelfennig 2007; Schimmelfennig/Sedelmeier 2004). Zur Erklärung des differenzierten Einflusses der EU-Integration auf die nationalen Arenen haben Europäisierungsforscher verschiedene Erklärungsmechanis- 
men entwickelt. Diese Mechanismen können nach ihren distinkten analytischen Ausgangspunkten unterschieden werden. Einige Autoren erachten den misfit zwischen europäischen und mitgliedstaatlichen institutionellen Strukturen als wesentlichen Katalysator für innerstaatlichen Wandel (Héritier et al. 1996; Börzel/Risse 2000, 2003). Andere konzentrieren sich auf die durch den europäischen Einfluss veränderten Opportunitätsstrukturen und die potenzielle Neuordnung von Akteurskonstellationen auf der nationalen Ebene (Schneider 2001). Eine dritte Gruppe von Wissenschaftlern verknüpft akteursbasierte und institutionalistische Erklärungen und kombiniert diese mit den der regulativen EU-Politik zugrunde liegenden Governance-Modi (Knill/Lenschow 1998, 2005; Bauer et al. 2007). Alle diese Mechanismen - wenn auch oftmals ohne klare Zuordnung und weniger elaboriert als in der Originalliteratur - finden sich in den Erklärungsversuchen von Regionalisierungs- und Dezentralisierungsprozessen in Mittelund Südosteuropa wieder.

Im Hinblick auf die Schaffung institutioneller Strukturen in Mittel- und Südosteuropa hat eine Reihe von Forschern dem Konditionalitätsregime der Europäischen Union zunächst großes Einflusspotenzial attestiert. Gelegentlich wurde sogar die Diffusion eines durch die Europäische Kommission geprägten Regionalmodells in die neuen Mitgliedstaaten prophezeit (kritisch dazu: Grabbe 2001, 2003; Jacoby 2004; Hughes 2003). Für eine solche Diffusion würde insbesondere die Machtasymmetrie zwischen der EU und den Bewerberstaaten sprechen. Durch die Definition konkreter Mitgliedschaftsbedingungen und das Setzen von Anreizen zur Umsetzung des acquis communautaire hätten der EU sehr einfache und effiziente Möglichkeiten zur Verfügung gestanden, die für den Beitrittsprozess gültigen Regeln unilateral festzulegen. Zweitens wurde damit gerechnet, dass die in den jährlichen Berichten über die Beitrittsvorbereitung der mittelund südosteuropäischen Länder zum Ausdruck gebrachte Präferenz der EU für ein bestimmtes Regionalmodell zu einem gewissen Grad zum Auftreten konvergenter regionaler Strukturen beitragen würde (Brusis 1999: 18). Drittens schätzen Wissenschaftler die im Rahmen diverser Unterstützungsprogramme gewährten finanziellen Anreize als einen wichtigen, die Diffusion eines bestimmten Regionalmodells begünstigenden Faktor ein. Da die EU die Kriterien definiert hat, welche die subnationalen Einheiten erfüllen müssen, um vor dem Beitritt finanzielle Unterstützung im Rahmen der Programme PHARE, SAPARD oder ISPA ${ }^{13}$ zu erhalten, haben Beitrittsländer - so die Argumentation - praktisch keine Alternative, als die von der EU festgesetzten institutionellen Standards für Regionalisierung und Dezentralisierung zu erfüllen (Yoder 2003).

Ein wesentlicher Vorteil der frühen Konditionalitätsstudien liegt darin, dass sie klar genug formuliert waren, um falsifiziert zu werden. Zwar konnte unter Zuhilfenahme des

13 PHARE ist ein Programm, das 1989 von der Europäischen Kommission initiiert wurde. Es hat die Unterstützung von Beitrittsländern beim Aufbau von Institutionen und einer funktionierenden Verwaltung sowie Finanzinvestitionen zum Ziel. Ursprünglich war das Programm für Polen und Ungarn aufgesetzt worden, es wurde jedoch mit der Zeit auf alle MOE-Beitrittsländer ausgedehnt. Im Jahr 2000 wurde PHARE ergänzt durch die Programme ISPA, welches auf den Umwelt- und den Transportsektor ausgerichtet ist, und SAPARD, dessen Fokus auf Landwirtschaft liegt. Für die Zeit von 2000 bis 2006 hat die EU zehn Milliarden Euro für PHARE bereitgestellt (http://europa.eu/scadplus/glossary/programme_phare_en.htm; Stand: 5.2.2008). 
Konditionalitätsarguments relativ präzise sowohl der Zeitrahmen als auch die Geschwindigkeit subnationaler Reformen vorhergesagt werden. Die erwartete Konvergenz in Bezug auf die institutionellen Strukturen hat sich aber nicht eingestellt. Folglich mussten die ursprünglichen Konditionalitätstheorien modifiziert werden. Angesichts der Tatsache, dass die Gestaltungskraft der EU offensichtlich viel geringer war als ursprünglich angenommen, setzen sich neuere Studien verstärkt mit den Gründen für die großen Unterschiede der beobachtbaren Ausprägungen subnationaler politischer Strukturen in den mittel- und südosteuropäischen Ländern auseinander. Damit rückten wieder nationale Faktoren wie z.B. die Politisierung subnationaler Reformen aufgrund parteipolitischer Machtkämpfe, funktionale Gründe ${ }^{14}$ und historische Erbschaften in den Blickpunkt der Forschung. Mit anderen Worten konvergierte nicht - wie ursprünglich angenommen die subnationale politische Realität unter dem Druck der EU-Konditionalität, sondern es näherten sich die Erklärungsprogramme der Transformations- und der Konditionalitätsforschung an, weil eingeräumt werden musste, dass die EU nur einer unter einer ganzen Reihe von einschlägigen Erklärungsfaktoren für die subnationale Dynamik in den mittel- und südosteuropäischen Ländern ist (Brusis 2002, 2005; Dieringer 2005; Futó et al. 2006; Hughes et al. 2004 a, 2004b; Jewtuchowicz et al. 2006; Lindstrom 2005; O'Dwyer 2006). Mit den Erweiterungsrunden von 2004 und 2007 und der damit einhergehenden nachlassenden Attraktivität der Konditionalitätstheorie für den mittelund südosteuropäischen Raum hat die „Entkopplung europäischer Politik von der innerstaatlichen Regionalpolitik" (Keating/Hooghe 2006: 282) weiter zugenommen.

Im Hinblick auf die methodische Herangehensweise der Konditionalitätsstudien ist festzustellen, dass die Anzahl komparativer Studien im Vergleich zum Transformationsansatz höher ausfällt und auch die zugrunde liegenden Forschungsdesigns ausgereifter sind. Oftmals werden zumindest grob gefasste Arbeitshypothesen entwickelt, die dann qualitativ in vergleichenden empirischen Analysen überprüft werden. Im Gegensatz zum Transformationsansatz ist die Verwendung von vergleichenden Fallstudiendesigns damit weitaus ausgeprägter. Jedoch konzentriert sich auch innerhalb des Konditionalitätsansatzes die überwältigende Mehrheit der Analysen auf die größeren mittel- und südosteuropäischen Länder, während Dynamiken in Bulgarien, Rumänien, den drei baltischen Staaten und Slowenien kaum systematisch untersucht werden (siehe Ausnahmen in Kettunen/Kungla 2005; Lindstrom 2005; von Breska/Brusis 1999).

Schließlich bleibt festzuhalten, dass der Konditionalitätsansatz den Einfluss der EU auf die Schaffung subnationaler institutioneller Strukturen in den mittel- und südosteuropäischen Ländern erforscht. Mit der Fokussierung auf Prozesse und strukturelle Ergebnisse nehmen die Konditionalitätsstudien - ebenso wie die Transformationsstudien eine Polity- und Politics-zentrierte Perspektive ein. Methodisch und theoretisch etwas stringenter als die Transformationsdebatte, hat der Konditionalitätsansatz doch einen Großteil seiner Attraktivität verloren, als die etwas zu euphorischen Erwartungen in Bezug auf die Konvergenz regionaler politischer Strukturen aufgegeben werden mussten. Ferner nahm die Relevanz des Konditionalitätsmechanismus mit dem EU-Beitritt

14 Aus einer funktionalistischen Perspektive betrachtet machte es keinen Sinn für kleinere Staaten wie Litauen, Lettland und Estland, eine voll institutionalisierte Ebene unterhalb der nationalen und oberhalb der lokalen Regierungsebene einzurichten (Kettunen/Kungla 2005). 
der mittel- und südosteuropäischen Länder weiter ab, weil damit faktisch die empirischen Fälle abhanden gekommen sind, die eine Relevanz des Mechanismus für die Regionalisierungsdebatte hätten zeigen können. Das Verdienst des Konditionalitätsparadigmas in der Debatte zur regionalen Entwicklung in den mittel- und südosteuropäischen Ländern liegt aber darin, dass durch die Synthese zweier Erklärungsfaktoren, nämlich unterschiedlicher Abstufungen supranationalen Drucks und diverser nationaler Kontextfaktoren, verschiedene subnationale Reaktionsmuster auf die Europäisierung bzw. Konditionalität unterscheidbar und beschreibbar wurden. Das wiederum erlaubte die Entwicklung differenzierter Hypothesen, die - zumindest im Prinzip - die in den mittel- und südosteuropäischen Ländern beobachtbare regionale Vielfalt einfangen können und damit als Ausgangspunkt für künftige Forschungsarbeiten in diesem Bereich dienen.

\subsection{Governance}

Mit der Umsetzung subnationaler Reformkonzepte und dem Vollzug des EU-Beitritts hat sich der politische Alltag in den mittel- und südosteuropäischen Ländern normalisiert. Die entweder neu geschaffenen oder wiederbelebten subnationalen Autoritäten haben ihre Arbeit aufgenommen. Daher verlieren die Fragen der subnationalen Institutionenbildung, welche die Regionalisierungsdebatte in den 1990er Jahren dominiert haben, an Bedeutung. Stattdessen interessieren sich Regionalisierungs- und Dezentralisierungsforscher stärker für die Implikationen subnationaler Beteiligung an der nationalen und europäischen Politikgestaltung (Baun/Marek 2006; Bauer 2004; Ferry 2007; Scherpereel 2007). Dieses im Entstehen begriffene Forschungsprogramm ist daher am besten als subnationaler Ableger der Governance-Debatte zu verstehen.

Zwei wesentliche Merkmale zeichnen diesen Ansatz aus. Im Gegensatz zum Transformations- bzw. zum Konditionalitätsansatz liegt der Fokus auf konkreten Policies und der Erfassung der empirischen Realität der Politikgestaltung in subnationalen Kontexten als solcher. Es wird also gerade nicht exklusiv nach Institutionenentwicklung (PolityDimension) oder allein nach den Effekten sich verändernder politischer Austauschbeziehungen (Politics-Dimension) gefragt. Diese Aspekte werden aber komplementär innerhalb dieser Analysen sehr wohl behandelt. Des Weiteren deutet sich an, dass mit dem Governance-Fokus auch die über den mittel- und südosteuropäischen Raum hinausgehenden komparativen Forschungsfragen an Bedeutung gewinnen. Somit findet eine Öffnung zur, wenn nicht gar eine Konvergenz mit der stark am europäischen Integrationskontext orientierten westeuropäischen Dezentralisierungs- und Regionalisierungsdebatte statt.

Seit sich das Interesse am Aufbau institutioneller Strukturen abgeschwächt und die Einsicht durchgesetzt hat, dass die Heterogenität der regionalen Autoritäten in den mittel- und südosteuropäischen Ländern ein dauerhaftes Merkmal darstellt, ist also die Untersuchung der differenzierten Einflussnahmemöglichkeiten der subnationalen Ebene auf nationale und supranationale Politikarenen in den Mittelpunkt wissenschaftlicher Forschung gerückt (Bauer/Kuppinger 2006; Futó et al. 2006; Grosse 2006; Marks et al. 2007; McMaster 2006; Paraskevopoulos et al. 2006; Scherpereel 2007). Fallstudien zur Tschechischen Republik oder zu Polen zeigen beispielsweise, dass der Dialog zwischen 
der subnationalen und der nationalen Ebene in Bezug auf regionale Mitwirkung intensiviert wurde und dass die subnationale Ebene insgesamt an Einfluss im innerstaatlichen Kontext gewonnen hat (Baun/Marek 2006; Scherpereel 2007). Die tschechischen und polnischen Erfahrungen können indes nicht vorbehaltlos auf alle mittel- und südosteuropäischen Länder übertragen werden. Denn in vielen anderen Ländern bleiben die Kapazitäten subnationaler Entitäten, sich in nationale und supranationale Politikgestaltung einzubringen, äußerst beschränkt (Grosse 2006; McMaster 2006). Dennoch zeichnet sich ein Trend dahingehend ab, dass subnationale Akteure in den größeren Staaten der ersten Beitrittsgruppe (Polen, Tschechische Republik, Slowakei, Ungarn) eher in der Lage sind, sich intensiver auf europäischer Ebene zu engagieren als die subnationalen politischen Akteure in den kleineren mittel- und südosteuropäischen Staaten (Bauer et al. 2007; Moore 2008).

Das Teilforschungsgebiet, auf dem sich die Konvergenz der westeuropäischen Regionalisierungsdebatte mit dem mittel- und südosteuropäischen Diskurs zu regionaler oder lokaler Politik am deutlichsten abzeichnet, ist das Feld der subnationalen Mobilisierung. Subnationale Mobilisierung im Kontext der EU wird als ,growing engagement of subnational governmental actors with the institutions and processes of EU policy-making“ definiert (Hooghe 1995). Die, wie man mittlerweile weiß, unerfüllte Erwartung früher Arbeiten in diesem Kontext war, dass die Stärkung subnationaler Akteure und Institutionen Legitimations- und Steuerungsgewinne für die Europäische Union bringen würde; und zwar unabhängig von oder sogar gegen die Interessen der zentralstaatlichen Ebene (Keating 2008). Mittlerweile ist aber klar, dass die von der EU unterstützte „Mobilisierung" der subnationalen Ebene nicht in allen Mitgliedstaaten gleichermaßen nachhaltige oder aus supranationaler Perspektive ,positive“ Reaktionen hervorgerufen hat (Benz/ Eberlein 1999; Bourne 2003; Fleurke/Willemse 2007). Stattdessen sind im EU-Vergleich enorme Unterschiede zwischen den subnationalen Entitäten in Bezug auf den jeweiligen Bedarf und den Umfang des jeweiligen Engagements in der europäischen Politikgestaltung feststellbar (Jeffery 2000; Hooghe 1995; Mols/Haslam 2008).

Diese Forschungsbemühungen haben eine Reihe von Erklärungsmechanismen generiert, die kulturelle, sozioökonomische oder institutionelle Faktoren für erhöhte Mobilisierungseffekte im subnationalen Raum verantwortlich machen. Diese Variablen haben sich als äußerst anschlussfähig im Hinblick auf die aufkommende subnationale Governance-Perspektive gezeigt; sie finden dort in unterschiedlichen Erklärungskombinationen regelmäßig Verwendung. Das Governance-Konzept bildet den Rahmen, um konkrete Politikresultate (Policies) als Produkt der Interaktion öffentlicher und privater Akteure auf verschiedenen Politikebenen zu erklären, wobei die unterschiedlichen Ausprägungen der Politikresultate im Zusammenhang mit der Verwendung von bestimmten Politikinstrumenten und den vorherrschenden Kontextfaktoren analysiert werden. Es ist ein Verdienst der Governance-Perspektive, in diesem Zusammenhang einen differenzierenden analytischen Zugriff auf das Phänomen der wachsenden politischen Bedeutung von Regionen als dem Produkt von Regionalisierungs- und Dezentralisierungsprozessen zu erlauben (John 2000; Roller/Sloat 2002). Die staatszentrierte Regionenforschung hat bislang nämlich die historische und sozioökonomische Basis von Regionalisierungstendenzen sowie absehbare Implikationen der Regionalisierung für den Zentralstaat (und das zumeist fokussiert auf die Beziehungen zwischen regionalen und zentralen Regie- 
rungen) in den Mittelpunkt ihrer Analysen gestellt. Die subnationale Governance-Forschung hingegen fragt - ohne freilich auf die traditionelle Sichtweise der Staat-Regionen-Beziehungen $\mathrm{zu}$ verzichten - insbesondere nach der Effizienz und Effektivität tatsächlich beobachtbaren regionalen Regierens. Sie stellt also auf konkrete Handlungssysteme, Akteurskonstellationen und Entscheidungsmodi ab (Sturm/Bauer 2009; Bauer/ Börzel 2009).

Das Forschungsprogramm der subnationalen Governance-Perspektive wird damit durch eine policy-analytische Agenda geprägt. Im Mittelpunkt stehen unterschiedlichen Politikergebnisse und weniger Fragen der Institutionenbildung, wie es bei den Transformations- oder den Konditionalitätsansätzen der Fall war (Sasse et al. 2006). Die subnationale Governance-Perspektive ist noch nicht konsolidiert. Daher sind Aussagen über vorherrschende Forschungsdesigns naturgemäß vorläufig. Qualitativ hochwertige Studien scheinen aber an Strategien der disziplinierten Fallstudienvergleiche, wie sie insbesondere von den Konditionalitätsforschern entwickelt wurden, anzuknüpfen (Baun/ Marek 2006; Ferry 2007; Scherpereel 2007). Eine Zusammenschau der zentralen Aussagen über die drei Forschungsprogramme gibt die folgende Tabelle.

Tabelle 4: Die drei Forschungsprogramme in der mittel- und südosteuropäischen Regionalisierungs- und Dezentralisierungsforschung

\begin{tabular}{|c|c|c|c|c|c|}
\hline $\begin{array}{l}\text { Forschungs- } \\
\text { programm }\end{array}$ & $\begin{array}{l}\text { Analyse- } \\
\text { fokus }\end{array}$ & $\begin{array}{l}\text { Erklärungs- } \\
\text { programm }\end{array}$ & $\begin{array}{l}\text { Mechanis- } \\
\text { mus }\end{array}$ & $\begin{array}{l}\text { Forschungs- } \\
\text { design }\end{array}$ & Zentrales Ergebnis \\
\hline $\begin{array}{l}\text { Systemtrans- } \\
\text { formation }\end{array}$ & $\begin{array}{l}\text { Systemtrans- } \\
\text { formation } \\
\text { auf der } \\
\text { subnationa- } \\
\text { len Ebene }\end{array}$ & $\begin{array}{l}\text { Historischer } \\
\text { Institutiona- } \\
\text { lismus }\end{array}$ & $\begin{array}{l}\text { Pfadab- } \\
\text { hängigkeit } \\
\text { (legacies) } \\
\text { Nationale } \\
\text { Politik }\end{array}$ & $\begin{array}{l}\text { Deskriptive } \\
\text { Fallstudien } \\
\text { (zumeist } \\
\text { Einzelfall- } \\
\text { studien) }\end{array}$ & $\begin{array}{l}\text { Regionalisierung und } \\
\text { Dezentralisierung als } \\
\text { Schritt in der „Rückkehr } \\
\text { der Länder nach Europa“; } \\
\text { Ergebnis: moderate } \\
\text { Regionalisierung bedingt } \\
\text { durch Pfadabhängigkeit }\end{array}$ \\
\hline $\begin{array}{l}\text { EU-Kondi- } \\
\text { tionalität }\end{array}$ & $\begin{array}{l}\text { Einfluss der } \\
\text { EU auf } \\
\text { Regionalisie- } \\
\text { rung und } \\
\text { Dezentrali- } \\
\text { sierung }\end{array}$ & $\begin{array}{l}\text { Europäisie- } \\
\text { rung }\end{array}$ & $\begin{array}{l}\text { EU-Kondi- } \\
\text { tionalitäts- } \\
\text { regime; } \\
\text { inner- } \\
\text { staatliche } \\
\text { Faktoren }\end{array}$ & $\begin{array}{l}\text { Analytische } \\
\text { Fallstudien } \\
\text { (verglei- } \\
\text { chende } \\
\text { Fallstudien) }\end{array}$ & $\begin{array}{l}\text { Prozess und Ergebnis der } \\
\text { Dezentralisierung und } \\
\text { Regionalisierung ist deter- } \\
\text { miniert durch innerstaat- } \\
\text { liche Faktoren (Regierungs- } \\
\text { präferenzen, parteipolitische } \\
\text { Positionen); } \\
\text { EU-Einfluss auf Timing } \\
\text { und Geschwindigkeit sub- } \\
\text { nationalen Institutions- } \\
\text { aufbaus }\end{array}$ \\
\hline $\begin{array}{l}\text { Subnationale } \\
\text { Governance }\end{array}$ & $\begin{array}{l}\text { Auswirkun- } \\
\text { gen differen- } \\
\text { zierten sub- } \\
\text { nationalen } \\
\text { Engagements } \\
\text { in der Politik- } \\
\text { gestaltung }\end{array}$ & $\begin{array}{l}\text { Eklektizis- } \\
\text { mus } \\
\text { Policy- } \\
\text { Analyse }\end{array}$ & $\begin{array}{l}\text { Verschiedene } \\
\text { subnationale } \\
\text { Ressourcen } \\
\text { (institutionell, } \\
\text { kulturell, } \\
\text { sozioökono- } \\
\text { misch) } \\
\text { (Subnationa- } \\
\text { ler) Parteien- } \\
\text { wettbewerb }\end{array}$ & $\begin{array}{l}\text { Ver- } \\
\text { gleichende } \\
\text { Fallstudien }\end{array}$ & $\begin{array}{l}\text { differenzierte Muster sub- } \\
\text { nationaler Mobilisierung } \\
\text { aufgrund unterschiedlicher } \\
\text { Ressourcen und bestimmter } \\
\text { nationaler Konstellationen }\end{array}$ \\
\hline
\end{tabular}




\section{Perspektiven}

Dieser Artikel hatte zum Ziel, Bilanz zur Dezentralisierungs- und Regionalisierungsforschung in den mittel- und südosteuropäischen Ländern im letzten Jahrzehnt zu ziehen, um die bisher entwickelten Argumente und die dabei gewonnenen Erkenntnisse zu systematisieren. Es konnten drei zentrale Forschungsansätze - Systemtransformation, EUKonditionalität und subnationale Governance - identifiziert werden, die sich bezüglich ihres Analysefokus, der vermuteten Erklärungsmechanismen und auch im Hinblick auf die gebräuchlichen Forschungsdesigns unterscheiden. Es ist deutlich geworden, dass Transformations- und Konditionalitätsstudien primär Institutionenemergenz und Institutionenwandel untersuchen und sich damit auf die Prozesse der Etablierung subnationaler Entitäten und die Schaffung von subnationalen institutionellen Strukturen konzentrieren. Studien, die dem Transformationsansatz zuzuordnen sind, nehmen insbesondere die unmittelbaren Auswirkungen des Zusammenbruchs der kommunistischen Regime in den Blick, wohingegen sich die Konditionalitätsstudien beobachtbaren Wandelprozessen als Ergebnis von Anpassungsdruck durch die Europäische Union widmen, die bei den Beitrittskandidaten die Umsetzung des acquis communautaire einfordert. Der aus unserer Sicht zentrale Punkt ist jedoch, dass die Relevanz dieser beiden Forschungsprogramme schwindet. Mit dem Vormarsch der Governance-Perspektive auch in diesem Teilbereich der Politikwissenschaft werden subnationale Fragen in den mittel- und südosteuropäischen Ländern zunehmend im Rahmen policy-analytischer Untersuchungen bearbeitet. Die Bandbreite dieser nun auf die subnationalen Konstellationen in Mittelund Südosteuropa angewandten Erklärungsstrategien ist groß, das Feld der unterschiedlichen Forschungsfragen also viel unübersichtlicher als in den relativ überschaubaren Debatten um Staatstransformation und EU-Konditionalität. Allerdings ist bemerkenswert, dass es sich faktisch überwiegend um eben jene Themen handelt, die noch vor einiger Zeit ausschließlich im Kontext von Analysen westeuropäischer Regionalstrukturen diskutiert wurden. Es kann also eine Konvergenz der Forschungsthemen zwischen dem west- und dem osteuropäischen Regionaldiskurs festgestellt werden. Diese Konvergenz darf als Ausdruck der „Normalisierung” der Regionalisierungs- und Dezentralisierungsforschung gelten. Forscher sind mittlerweile weniger am subnationalen Geschehen als Ausprägung spezifisch mittel- und südosteuropäischer Problematiken interessiert; vielmehr werden Fallspezifika aus diesen Ländern mit westeuropäischen verglichen. Insbesondere, wenn es um die Analyse der subnationalen Dimension gegenwärtiger Politikgestaltungsprozesse geht, erhöhen sich damit die Chancen, über die Auswahl von Fallbeispielen aus ganz Europa und über die Verwendung vergleichender Forschungsdesigns zu überzeugenderen Ergebnissen zu kommen. Vorbedingung dafür ist es, Ost- und Westeuropa nicht mehr als separate Einheiten zu interpretieren. Das Forschungsprogramm der subnationalen Governance - gerade auch als Bestandteil einer im Entstehen begriffenen Theorie von Multilevel-Governance (Hooghe/Marks 2001) - wird also voraussichtlich die Regionalisierungs- und Dezentralisierungsforschung in der erweiterten EU künftig immer stärker prägen. 


\section{Literatur}

Alesina, Albert, und Enrico Spolaore. 2003. The Size of Nations. Cambridge, MA: MIT Press.

Altmann, Franz L. 2002. Regionale Kooperation in Südosteuropa. Organisationen, Pläne, Erfahrungen. Südosteuropa. Zeitschrift für Gegenwartsforschung 51: 266-288.

*Bachtler, John, Ruth Downes und Grzegorz Gorzelak (Hrsg.). 2000. Transition, Cohesion and Regional Policy in Central and Eastern Europe. Aldershot: Ashgate.

*Bafoil, François. 1999. Post-Communist Borders and Territories: Conflicts, Learning and RuleBuilding in Poland. International Journal of Urban and Regional Research 23: 567-582.

*Bailey, David, und Lisa De Propris. 2002. EU Structural Funds, Regional Capabilities and Enlargement: Towards Multi-level Governance? Journal of European Integration 24: 303-324.

*Balás, Gabór, und Jószef Hegedüs. 2003. Local Self-Government and Decentralization in Hungary. Budapest: Friedrich-Ebert-Stiftung.

Barna, Claudia, Hristo Panchugov, Marius Tatar und Vladimir Todorakov. 2005. Europeanization of Regions and the Partnership Principle: The Bulgarian Case. Südosteuropa. Zeitschrift für Gegenwartsforschung 53: 557-572.

*Batt, Judy. 2002a. Reinventing Banat. Regional and Federal Studies 12: 178-202.

*Batt, Judy. 2002b. Transcarpathia: Peripheral Region at the Centre of Europe. Regional and Federal Studies 12: 155-177.

Batt, Judy. 2002c. Introduction: Region, State and Identity in Central and Eastern Europe. In: Batt/Wolczuk (Hrsg.) 2002, 1-14.

*Batt, Judy. 2003. The Impact of EU Enlargement on Regions on the EU's New Eastern Border. Geneva: United Nations Economic Commission for Europe.

*Batt, Judy, und Kataryna Wolczuk (Hrsg.). 2002. Region, State and Identity in Central and Eastern Europe. London und Portland: Frank Cass.

Bauer, Michael W. 2004. Erweiterungsdynamik? Das Europa der Regionen. Osteuropa. Zeitschrift für Gegenwartsfragen des Ostens 54: 160-179.

Bauer, Michael W., und Tanja A. Börzel. 2009. Regions and the EU. In: Michael Zürn, Henrik Enderlein und Sonja Wälti (Hrsg.). Handbook on Multilevel Governance. Cheltenham: Edward Elgar [im Erscheinen].

Bauer, Michael W., Christoph Knill und Diana Pitschel. 2007. Differential Europeanization in Eastern Europe: The Impact of Diverse EU Regulatory Governance Patterns. Journal of European Integration 29: 405-424.

Bauer, Michael W., und Jutta Kuppinger. 2006. Subnationale Autonomie und EU-Regionalförderung. Polen und Tschechien im Vergleich. Osteuropa. Zeitschrift für Gegenwartsfragen des Ostens 56: 105-115.

Bauer, Michael W., und Diana Pitschel. 2006. Europäisierung als Erklärung von Dezentralisierung und Regionalisierung in Mittel- und Osteuropa - Mehr Fragen als Antworten. In: Jahrbuch des Föderalismus 2006. Föderalismus, Subsidiarität und Regionen in Europa, hrsgg. v. Europäischen Zentrum für Föderalismus-Forschung Tübingen. Baden-Baden: Nomos, 44-56.

Bauer, Michael W., und Diana Pitschel. 2007. Akteurspräferenzen: Schlüssel zur Erklärung subnationaler Mobilisierung. In: Jahrbuch des Föderalismus 2007. Föderalismus, Subsidiarität und Regionen in Europa, hrsgg. v. Europäischen Zentrum für Föderalismus-Forschung Tübingen. Baden-Baden: Nomos, 74-85.

Bauer, Michael W., Diana Pitschel und Ansgar Schäfer. 2007. The Debate on New Modes of Governance and the Subnational Dimension. Vortrag anlässlich des internationalen Seminars „New Modes of Governance in the New and Old Member States: Similarities and Differences" (NEWGOV), 26. Oktober 2007, Warschau, Sektion I: Emergence of the New Modes of Governance and Its Impact in Terms of Legitimacy and Effectiveness.

*Baun, Michael. 2002. EU Regional Policy and the Candidate States: Poland and the Czech Republic. Journal of European Integration 24: 261-80. 
*Baun, Michael, und Dan Marek. 2006. Regional Policy and Decentralization in the Czech Republic. Regional and Federal Studies 16: 409-428.

Benz, Arthur, und Burkard Eberlein. 1999. The Europeanization of Regional Politics. Patterns of Multi-Level Governance. Journal of European Public Policy 6: 329-348.

Bessenyey-Williams, Margit. 2007. On Europe's Edge: Changing Borders in Central and Eastern Europe. In: Katalin Fábián (Hrsg.). Globalization: Perspectives from Central and Eastern Europ. Amsterdam/London: JAI, 115-150.

*Bialasiewicz, Luiza. 2002. Upper Silesia: Rebirth of Regional Identity in Poland. Regional and Federal Studies 12: 111-32.

*Bitušíkóva, Alexandra. 2002. Slovakia: An Anthropological Perspective on Regional Reform. Regional and Federal Studies 12: 41-64.

Börzel, Tanja A., und Thomas Risse. 2000. When Europe Hits Home: Europeanization and Domestic Change. European Integration Online Papers 4 [http://eiop.or.at/eiop/texte/2000015a.htm.; 30. 11.2007].

Börzel, Tanja A., und Thomas Risse. 2003. Conceptualizing the Domestic Impact of Europe. In: Kevin Featherstone und Claudio M. Radaelli (Hrsg.). The Politics of Europeanization. Oxford: Oxford University Press, 57-81.

Bookman, Milica Z. 1993. The Economics of Secession. New York: Palgrave Macmillan.

Bourne, Angela. 2003. The Impact of European Integration on Regional Power. Journal of Common Market Studies 41: 597-620.

Braun, Aurel. 2000. All Quiet on the Russian Front? Russia, Its Neighbors, and the Russian Diaspora. In: Michael Mandelbaum (Hrsg.). The New European Diasporas: National Minorities and Conflict in Eastern Europe. New York: The Council on Foreign Relations, 81-158.

*von Breska, Erik, und Martin Brusis. 1999. Central and Eastern Europe on the Way into the European Union: Reforms of Regional Administration in Bulgaria, the Czech Republic, Estonia, Hungary, Poland and Slovakia. München: Centrum für Angewandte Politikforschung.

Brubaker, Rogers. 1996. Nationalism Reframed: Nationhood and the National Question in the New Europe. Cambridge: Cambridge University Press.

Brusis, Martin. 1999. Re-Creating the Regional Level in Central and Eastern Europe: An Analysis of Administrative Reforms in Six Countries'. In: Eric von Breska und Martin Brusis (Hrsg.). Central and Eastern Europe on the Way into the European Union: Reforms of Regional Administration in Bulgaria, the Czech Republic, Estonia, Hungary, Poland and Slovakia. München: Centrum für Angewandte Politikforschung, 1-22.

*Brusis, Martin. 2002. Between EU Requirements, Competitive Politics and National Traditions: Re-creating Regions in the Accession Countries of Central and Eastern Europe. Governance: An International Journal of Policy, Administration and Institutions 15: 531-559.

Brusis, Martin. 2003. Regionalisierungsprozesse in Mittel- und Osteuropa. Institutionelle und fiskalische Aspekte regionaler Autonomie. Osteuropa-Wirtschaft 48: 309-332.

*Brusis, Martin. 2005. The Instrumental Use of European Union Conditionality: Regionalization in the Czech Republic and Slovakia. East European Politics and Societies 19: 291-316.

Brusis, Martin, und Viktor Nižnanský. 1997. Die slowakische Verwaltungs- und Gebietsreform unter minderheitspolitischem Aspekt. Südosteuropa. Zeitschrift für Gegenwartsforschung 46: 197-212.

*Bryson, Phillip J., und Gary C. Cornia. 2000. Fiscal Decentralisation in Economic Transformation: The Czech and Slovak Cases. Europe-Asia Studies 52: 507-522.

*Bryson, Phillip J., und Gary C. Cornia. 2004. Public Sector Transition in Post-communist Economies: The Struggle for Fiscal Decentralisation in the Czech and Slovak Republic. PostCommunist Economies 16: 265-283.

*Bryson, Phillip J., Gary C. Cornia und Gloria C. Wheeler. 2004. Fiscal Decentralisation in the Czech and Slovak Republics: A Comparative Study of Moral Hazard. Environment and Planning C: Government and Policy 22: 103-113. 
Buhrova, Iryna. 2004. Einheit über Vielfalt? Regionen im Zentralstaat. Osteuropa 54: 39-56.

Caplan, Richard, und John Feffer. 1996. Europe's New Nationalism: States and Minorities in Conflict. Oxford: Oxford University Press.

Caramani, Daniele. 2003. State Administration and Regional Construction in Central Europe: A Comparative-Historical Perspective. In: Keating/Hughes (Hrsg.) 2003, 21-50.

Cini, Michelle. 2003. European Union Politics. Oxford: Oxford University Press.

Committee of Regions (CoR). 2005. CoR-Studies 1/2005 [http://www.cor.europa.eu/document/ documents/decentralisationEN.pdf; 05.02.2008].

Connor, Walker. 1994. Ethnonationalism: The Quest for Understanding. Princeton: Princeton University Press.

*Czernielewska, Malgorzata, Christos J. Paraskevopoulos und Jacek Szlachta. 2004. The Regionalization Process in Poland: An Example of "Shallow" Europeanization? Regional and Federal Studies 14: 461-493.

Dieringer, Jürgen. 2005. Demokratisierung, Ökonomisierung und Europäisierung: Dezentralisierungsprozesse in Ostmitteleuropa. Südosteuropa. Zeitschrift für Gegenwartsforschung 53: 483-499.

Dieringer, Jürgen, und Ionel-Sorin Moisa. 2001. Öffentliche Verwaltung versus Regionalpolitik? Die Europäisierung der Regionalstruktur Ungarns und Rumäniens. Südosteuropa. Zeitschrift für Gegenwartsforschung 50: 537-560.

*Dimitrova, Antoaneta L. (Hrsg.). 2004. Driven to Change. The European Union's Enlargement Viewed from the East. Manchester/New York: Manchester University Press.

*Dobre, Ana M. 2005. The Dynamics of Europeanisation, Regionalisation and Regionalism: The Romanian Case of Regional Re-construction. Südosteuropa. Zeitschrift für Gegenwartsforschung 53: 540-556.

Esman, Milton J. (Hrsg.). 1977. Ethnic Conflict in the Western World. Ithaca: Cornell University Press.

Europäisches Zentrum für Föderalismus-Forschung Tübingen (EZFF) (Hrsg.). 2004-2007. Jahrbuch des Föderalismus. Baden-Baden: Nomos.

*Faina, J. Andrés, und Jesús López-Rodríguez. 2001. The Regional Policy of the European Union and the Enlargement Process to Central and Eastern European Countries. Exeter: University of Exeter.

*Ferry, Martin. 2003. The EU and Recent Regional Reform in Poland. Europe-Asia-Studies 55, 1097-1116.

*Ferry, Martin. 2007. From Government to Governance: Polish Regional Development Agencies in a Changing Regional Context. East European Politics and Societies 21: 447-474.

*Ferry, Martin, und Irene McMaster. 2005. Implementing Structural Funds in Polish and Czech Regions. Convergence, Variation, Empowerment? Regional and Federal Studies 15: 19-39.

Fleurke, Frederik, und Rolf Willemse. 2007. Effects of the European Union on Sub-National Decision-making: Enhancement or Constriction? Journal of European Integration 29: 69-88.

*Fowler, Brigid. 2002. Hungary: Patterns of Political Conflict over Territorial-administrative Reform. Regional and Federal Studies 12: 15-40.

Frenkel, Michael, und Christiane Nickel. 2005. How Symmetric are the Shocks and the Shock Adjustment Dynamics between the Euro Area and Central and Eastern Europe? Journal of Common Market Studies 43: 53-74.

Futó, Péter, Ilona P. Kovács und Tamás Fleischer. 2006: Governance in Regional and Environmental Policies in Hungary: Challenges of Europeanisation and Adaptation. In: Paraskevopoulos/ Getimis/Rees (Hrsg.) 2006, 107-135.

Gabel, Matthew, Guy D. Whitten. 1997. Economic Conditions, Economic Perceptions and Public Support for European Integration. Political Behaviour 19: 81-96.

Gerner, Kristian. 2005: The Role of the Baltic States, Poland and Hungary in the New Europe. In: Sergio Ortino, Mitja Zagar und Vojtech Mastny (Hrsg.). The Changing Faces of Federal- 
ism. Institutional Reconfiguration in Europe from East to West. Manchester: Manchester University Press, 63-84.

*Gorzelak, Grzegorz. 1998. Regional and Local Potential for Transformation in Poland. Warschau: European Institute for Local and Regional Development.

*Grabbe, Heather. 2001. How Does Europeanization Affect CEE Governance? Conditionality, Diffusion and Diversity. Journal of European Public Policy 8: 1013-1031.

Grabbe, Heather. 2003. Europeanization Goes East: Power and Uncertainty in the EU Accession Process. In: Kevin Featherstone, und Claudio M. Radaelli (Hrsg.). The Politics of Europeanization. Oxford: Oxford University Press, 303-327.

*Grosse, Tomasz G. 2006. An Evaluation of the Regional Policy System in Poland: Challenges and Threats Emerging from Participation in the EU's Cohesion Policy. European Urban and Regional Studies 13: 151-165.

*Grúber, Károly. 2002. Globalisation, Regions, Nations and Integration: Central and Western European Perspectives. Perspectives on European Politics and Societies 3: 199-228.

Hall, Peter A. 1986. Governing the Economy: The Politics of State Intervention in Britain and France. New York: Oxford University Press.

Hall, Peter A., und Rosemary C. R. Taylor. 1996. Political Science and the Three New Institutionalisms. Political Studies 44: 936-957.

Harvie, Christopher. 1994. The Rise of Regional Europe. London: Routledge.

Héritier, Adrienne. 2002. New Modes of Governance in Europe: Policy-Making without Legislating? In: Dies. (Hrsg.). Common Goods: Reinventing European and International Governance. Lanham, MD: Rowman \& Littlefield, 185-206.

Héritier, Adrienne, Christoph Knill und Susanne Mingers. 1996. Ringing the Changes in Europe: Regulatory Competition and the Transformation of the State. Berlin: De Gruyter.

Hix, Simon, und Klaus H. Goetz. 2000. Europeanised Politics? European Integration and National Political Systems. London: Frank Cass.

Hooghe, Liesbet. 1995. Subnational Mobilisation in the European Union. West European Politics 18: 175-198.

Hooghe, Liesbet, und Gary Marks. 2001. Multi-level Governance and European Integration. Lanham, MD: Rowman \& Littlefield.

*Horváth, Tamás M. 1997. Decentralization in Public Administration and Provision of Services: an East-Central European View. Environment and Planning C: Government and Policy 15: 161-175.

*Horváth, Tamás M. 2000. Decentralization: Experiments and Reforms. Budapest: Local Government and Public Service Reform Initiative, Open Society Institute.

Hughes, James. 2003. Regional Convergence and Divergence in an Enlarged EU. In: Keating/ Hughes (Hrsg.) 2003, 183-192.

Hughes, James, Gwendolyn Sasse und Claire E. Gordon. 2001. Enlargement and Regionalization: The Europeanization of Local and Regional Governance in CEE States. In: Helen Wallace (Hrsg.). Interlocking Dimensions of European Integration. Basingstoke: Palgrave, 145-178.

Hughes, James, Gwendolyn Sasse und Claire E. Gordon. 2002. Saying "Maybe" to the "Return to Europe". Elites and the Political Space for Euroscepticism in Central and Eastern Europe. European Union Politics 3: 327-355.

Hughes, James, Gwendolyn Sasse und Claire E. Gordon. 2003. EU Enlargement, Europeanisation and the Dynamics of Regionalisation in the CEECs. In: Keating/Hughes (Hrsg.) 2003, 69-88.

*Hughes, James, Gwendolyn Sasse und Claire E. Gordon. 2004a: Europeanization and Regionalization in the EU's Enlargement to Central and Eastern Europe. Houndmills: Palgrave Macmillan.

*Hughes, James, Gwendolyn Sasse und Claire E. Gordon. 2004b. Conditionality and Compliance in the EU's Eastward Enlargement: Regional Policy and the Reform of Subnational Government. Journal of Common Market Studies 42: 523-551. 
*Jacoby, Wade. 2004. The Enlargement of the European Union and NATO: Ordering from the Menu in Central and Eastern Europe. Cambridge: Cambridge University Press.

Illner, Michal. 1997a. Regional Structure and Post-communist Transformation - the Case of the Czech Republic. In: Jiri Musil und Wendelin Strubelt (Hrsg.). Räumliche Auswirkungen des Transformationsprozesses in Deutschland und bei den östlichen Nachbarn. Opladen: Leske + Budrich, 29-44.

Illner, Michal. 1997b. Territorial Decentralization - a Stumbling Block of Democratic Reforms in East-Central Europe? Polish Sociological Review I 117: 23-45.

Illner, Michal. 2002. Multilevel Government in Three East Central European Candidate Countries and its Reforms after 1989. EUI Working Paper, RSC No. 2002 /7. S. Domenico di Fiesole: European University Institute,

Immergut, Ellen M. 1998. The Theoretical Core of the New Institutionalism. Politics \& Society 26: 5-34.

Jeffery, Charlie. 2000. Sub-National Mobilization and European Integration: Does it Make Any Difference? Journal of Common Market Studies 38: 1-23.

Jewtuchowicz, Aleksandra, und Malgorzata Czernielewska-Rutkowska. 2006. Between Institutional Legacies and the Challenges of Europeanization: Governance and Learning in Regional and Environmental Policies in Poland. In: Paraskevopoulos/Getimis/Rees (Hrsg.) 2006, 137-176.

John, Peter. 2000. Europeanisation of Subnational Governance. Urban Studies 37: 877-894.

Jolly, Seth K. 2007. The Europhile Fringe: Regionalist Party Support for European Integration. European Union Politics 8: 109-130.

Keating, Michael. 1998. The New Regionalism in Western Europe: Territorial Restructuring and Political Change. Cheltenham: Edward Elgar.

Keating, Michael. 2008. Thirty Years of Territorial Politics. West European Politics 31: 60-81.

Keating, Michael, und Liesbet Hooghe. 2006. Bypassing the Nation State? Regions and the EU Policy Process. In: Jeremy Richardson (Hrsg.). European Union. Power and Policy-Making. London: Routledge, 216-229.

*Keating, Michael, und James Hughes (Hrsg.). 2003. The Regional Challenge in Central and Eastern Europe. Territorial Restructuring and European Integration. Brüssel: Peter Lang.

*Kettunen, Pekka, und Tarvo Kungla. 2005. Europeanization of Sub-national Governance in Unitary States: Estonia and Finland. Regional and Federal Studies 15: 353-378.

*Keune, Maarten. 1998. Regional Development and Employment Policy: Lessons from Central and Eastern Europe. Geneva: ILO.

*Kimball, Jonathan D. (Hrsg.). 1999. The Transfer of Power: Decentralization in Central and Eastern Europe. Budapest: The Local Government and Public Service Reform Initiative.

*Kirchner, Emil J. (Hrsg.). 1999. Decentralization and Transition in the Visegrad. Houndmills: Palgrave Macmillan.

Knill, Christoph, und Andrea Lenschow. 1998. Coping with Europe: The Impact of British and German Administrations on the Implementation of EU Environmental Policy. Journal of European Public Policy 5: 595-615.

Knill, Christoph, und Andrea Lenschow. 2005. Coercion, Competition and Communication: Different Approaches of European Governance and their Impact on National Institutions. Journal of Common Market Studies 43: 581-604.

Kohler-Koch, Beate. 1995. Regions as Political Actors in the Process of European Integration. A Research Design. Mannheimer Zentrum für Europäische Sozialforschung (MZES), Arbeitspapier Nr. 9, Mannheim.

Kohler-Koch, Beate. 1998. Regionale Leistungskraft und regionale Nutzenbilanz. In: Dies. (Hrsg.). Interaktive Politik in Europa: Regionen im Netzwerk der Integration. Opladen: Leske + Budrich, 125-152. 
Kovrig, Bennett. 2000. Partitioned Nation: Hungarian Minorities in Central Europe. In: Michael Mandelbaum (Hrsg.). The New European Diasporas: National Minorities and Conflict in Eastern Europe. New York: The Council on Foreign Relations, 19-80.

Kymlicka, Will. 2000. Nation-Building and Minority Rights: Comparing West and East. Journal of Ethnic and Migration Studies 26: 183-212.

Lindstrom, Nicole. 2005. Europeanization and Sub-national Governance in Slovenia. Südosteuropa. Zeitschrift für Gegenwartsforschung 53: 500-519.

*Marcou, Gérard (Hrsg.). 2002. Regionalization for Development and Accession to the European Union: A Comparative Perspective. Budapest: Local Government and Public Service Reform Initiative, Open Society Institute.

*Marek, Dan, und Michael Baun. 2002. The EU as Regional Actor: The Case of the Czech Republic. Journal of Common Market Studies 40: 895-919.

Marks, Gary. 1992. Structural Policy in the European Community. In: Alberta M. Sbragia (Hrsg.). Euro-Politics: Institutions and Policy-Making in the "New" European Community. Washington, D. C.: Brookings Institution, 191-224.

Marks, Gary, Liesbet Hooghe und Arjan Schakel. 2007. Patterns of Regional Authority, Paper presented at the European Union Studies Association, 17.-19. Mai 2007. Montreal.

Marks, Gary, François Nielsen, Leonard Ray und Jane Salk. 1996. Competencies, Cracks and Conflicts: Regional Mobilization in the European Union. In: Gary Marks, Fritz W. Scharpf, Philippe Schmitter und Wolfgang Streeck (Hrsg.). Governance in the European Union. London: SAGE, 40-63.

Mastenbroek, Ellen, und Michael Kaeding. 2006. Europeanization Beyond the Goodness of Fit: Domestic Politics in the Forefront. Comparative European Politics 4: 331-354.

*Mastny, Vojtech. 2000. The Historical Experience of Federalism in East Central Europe. East European Politics and Societies 14: 64-96.

*McMaster, Irene. 2006. Czech Regional Development Agencies in a Shifting Institutional Landscape. Europe-Asia Studies 58: 347-370.

Meegan, Edward J., und Vasil Hudák. 1999. Regional Policy Goes East: Essays on Trends and Lessons Learned for Regional Development Policy in Central and Eastern Europe. Prag: EastWest Institute.

Mols, Frank, und S. Alexander Haslam. 2008. Understanding EU Attitudes in Multi-Level Governance Contexts: A Social Identity Perspective. West European Politics 31: 442-463.

Moore, Carolyn. 2008. Beyond Conditionality? Regions from the New EU Member States and their Activities in Brussels. Comparative European Politics 6: 212-234.

Mudde, Cas. 2005. Racist Extremism in Central and Eastern Europe. East European Politics and Societies 19: 161-184.

*Myant, Martin, und Simon Smith. 2006. Regional Development and Post-Communist Politics in a Czech Region. Europe-Asia Studies 58, 147-168.

*O'Dwyer, Conor. 2006. Reforming Regional Governance in East Central Europe: Europeanization or Domestic Politics as Usual? East European Politics and Societies 20: 219-253.

*Ortino, Sergio, Mitja Zagar und Mitja Vojtech (Hrsg.). 2005. The Changing Faces of Federalism. Institutional Reconfiguration in Europe from East to West. Manchester: Manchester University Press.

*Pamfil, Claudia. 2003. Evolving Intergovernmental Relations for Effective Development in the Context of Regionalization. Budapest: Central European University Press.

*Pálné Kovács, Ilona, Christos J. Paraskevopoulos und Gyula Horváth. 2004. Institutional "Legacies" and the Shaping of Regional Governance in Hungary. Regional and Federal Studies 14: 430-460.

*Paraskevopoulos, Christos J., und Robert Leonardi. 2004. Introduction: Adaptational Pressures and Social Learning in European Regional Policy - Cohesion (Greece, Ireland and Portugal) vs. CEE (Hungary, Poland) Countries. Regional and Federal Studies 14: 315-354. 
*Paraskevopoulos, Christos J., Panayotis Getimis und Nicholas Rees (Hrsg.). 2006. Adapting to EU Multi-level Governance: Regional and Environmental Policies in Cohesion and CEE Countries. Aldershot: Ashgate.

*Péteri, Gabor (Hrsg.). 2003. Mastering Decentralisation and Public Administration Reforms in Central and Eastern Europe. Budapest: Central European University Press.

*Petrakos, Giōrgos C. 1997. The Regional Structure of Albania, Bulgaria and Greece: Implications for Cross-border Cooperation and Development. European Urban and Regional Studies 4: 195-210.

Pieper, Karin. 2006. Regionalpolitik in Ungarn und Polen. Wiesbaden: VS Verlag für Sozialwissenschaften.

Pogátsa, Zoltán. 2005. Aborted Regionalisation in Hungary. Südosteuropa. Zeitschrift für Gegenwartsforschung 53: 520-539.

Roller, Elisa, und Amanda Sloat. 2002. The Impact of Europeanisation on Regional Governance: A Study of Catalonia and Scotland. Public Policy and Administration 17: 68-86.

*Rose, Jürgen, und Johannes Traut. 2001. Federalism and Decentralisation: Perspectives for the Transformation Process in Eastern and Central Europe. Münster: LIT.

*Sadurski, Wojciech, Adam Czarnota und Martin Krygier (Hrsg.). 2006. Spreading Democracy and the Rule of Law? The Impact of EU Enlargement on the Rule of Law, Democracy and Constitutionalism in Post-Communist Legal Orders. Dordrecht: Springer.

*Sasse, Gwendolyn, und James Hughes. 2004. Integration mit Tiefgang? Regionalisierung in Ungarn und Polen. Osteuropa 54: 180-194.

Sasse, Gwendolyn, James Hughes und Claire E. Gordon. 2006. Subnational Governance in Central and Eastern Europe: Between Transition and Europeanization. In: Sadurski/Czarnota/ Krygier (Hrsg.) 2006, 121-147.

*Scherpereel, John A. 2007. Sub-National Authorities in the EU's Post-Socialist States. European Integration 29: 23-46.

Schimmelfennig, Frank, 2007. Europeanization Beyond Europe. Living Reviews in European Governance 2 [http://www.livingreviews.org//reg-2007-1.; 20.09.2007].

Schimmelfennig, Frank, und Ulrich Sedelmeier. 2004. Governance by Conditionality: EU Rule Transfer to the Candidate Countries of Central and Eastern Europe. Journal of European Public Policy 11: 661-679.

*Schimmelfennig, Frank, und Ulrich Sedelmeier (Hrsg.). 2005. The Europeanization of Central and Eastern Europe. Ithaca, N. Y.: Cornell University Press.

Schneider, Volker. 2001. Europeanization and the Redimensionalization of the Public Sector: Telecommunication in Germany, France and Italy. In: Maria G. Cowles, James Caporaso und Thomas Risse (Hrsg.). Transforming Europe. Europeanisation and Domestic Change. Ithaca, N. Y.: Cornell University Press, 60-78.

*Smith, Simon, und Martin Myant. 2003. EU Accession, Regional Policy and Economic Development in East Central Europe. Paisley: University of Paisley Press.

Soós, Gábor, Gábor Tóka und Glen Wright (Hrsg.). 2002. The State of Local Democracy in Central Europe. Budapest: Local Government and Public Sector Reform Institute.

Sturm, Roland, und Michael W. Bauer. 2009. Governance und Regionen - die theoretische Debatte. In: Roland Sturm und Jürgen Dieringer (Hrsg.). Regional Governance in der Europäischen Union. Opladen: Barbara Budrich [im Erscheinen].

*Sturm, Roland und Jürgen Dieringer. 2005. The Europeanization of Regions in Eastern and Western Europe: Theoretical Perspectives. Regional and Federal Studies 15: 279-294.

*Szemlér, Tamás. 2000. Regional Development and the EU Pre-Accession and Structural Funds: Trends and Possible Courses of Action. Budapest: Hungarian Academy of Science.

Szücs, Stefan (Hrsg.). 2006. Local Elites, Political Capital and Democratic Development: Governing Leaders in Seven European Countries. Wiesbaden: VS Verlag für Sozialwissenschaften. 
Tägil, Sven (Hrsg.). 1999. Regions in Central Europe: The Legacy of History. London: Hurst.

*Tatur, Melanie (Hrsg.). 2004. The Making of Regions in Post-Socialist Europe - The Impact of Culture, Economic Structure and Institutions. Case Studies from Poland, Hungary, Romania and Ukraine. Wiesbaden: VS Verlag für Sozialwissenschaften.

Thelen, Kathleen, und Sven Steinmo. 1992. Historical Institutionalism in Comparative Politics. In: Sven Steinmo, Kathleen Thelen und Frank Longstreth (Hrsg.). Structuring Politics. Historical Institutionalism in Comparative Analysis. Cambridge: Cambridge University Press, $1-32$.

Van Houten, Pieter. 2003. Globalization and Demands for Regional Autonomy in Europe. In: Miles Kahler und David A. Lake (Hrsg.). Governance in a Global Economy. Political Authority in Transition. Princeton: Princeton University Press, 110-135.

*Yilmaz, Serdar, Joszef Hegedüs und Michael Bell (Hrsg.). 2003. Subnational Data Requirements for Fiscal Decentralization: Case Studies from Central and Eastern Europe. Washington, D. C.: World Bank.

*Yoder, Jennifer A. 2003. Decentralisation and Regionalisation after Communism: Administrative and Territorial Reform in Poland and the Czech Republic. Europe-Asia Studies 55: 263-286.

*Yoder, Jennifer A. 2007. Leading the Way to Regionalization in Post-Communist Europe: An Examination of the Process and Outcomes of Regional Reform in Poland. East European Politics and Societies 21: 424-446.

Zloch, Stephanie. 2000. Polens neue Regionen auf dem Weg in die EU. Osteuropa 50, 367-381. 